\title{
Silicon-Based Single Quantum Dot Emission in the Telecoms C-Band
}

Jonathan R. Orchard, ${ }^{\dagger}$ Chris Woodhead, ${ }^{\ddagger}$ Jiang Wu, ${ }^{\S}{ }^{\circledR}$ Mingchu Tang, ${ }^{\S}$ Richard Beanland, ${ }^{\|}$Yasir Noori, Huiyun Liu, ${ }^{\S}$ Robert J. Young, ${ }^{\ddagger}$ and David J. Mowbray, ${ }^{*}+(0)$

${ }^{\dagger}$ Department of Physics, University of Sheffield, Hicks Building, Hounsfield Road, Sheffield S3 7RH, United Kingdom

${ }^{\ddagger}$ Department of Physics, University of Lancaster, Lancaster LA1 4YB, United Kingdom

${ }^{\S}$ Department of Electronic and Electrical Engineering, University College London, Torrington Place, London WC1E 7JE, United Kingdom

"Department of Physics, University of Warwick, Gibbet Hill Road, Coventry CV4 7AL, United Kingdom

\section{Supporting Information}

ABSTRACT: We report the observation of single quantum dot (QD) emission in the telecoms C-band (1530-1565 nm) from an InAs QD structure grown on a Si substrate. A large red-shift of the emission is achieved by capping InAs QDs with a thin $\mathrm{GaAsSb}$ layer. Sharp lines, representing emission from single QDs, are observed out to wavelengths as long as $1540 \mathrm{~nm}$. Comparison is made to the optical properties of a nominally identical active region structure grown on a GaAs substrate. Single QD emission from a Si-based system at $1500 \mathrm{~nm}$ has the potential for single photon sources compatible with current optical fibers and reduced complexity of integration with drive electronics.

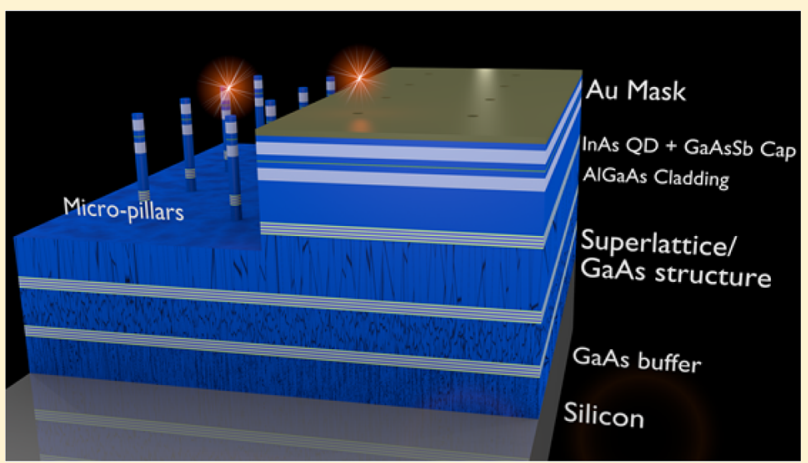

KEYWORDS: single-photon sources, quantum dots, Si substrate, C-band, III-V semiconductors, GaAsSb

$\mathrm{T}^{\mathrm{k}}$ he unique, discrete electronic density of states exhibited by semiconductor quantum dots (QDs) provides many applications for both conventional and novel light emitters. An example of the latter is the single photon source, with application in the field of quantum cryptography. Here a single $\mathrm{QD}$ is loaded optically or electrically with a quasi-random number of excitons. Because the photon energy resulting from a recombining exciton is dependent on the number of excitons remaining in the dot, subsequent radiative decay of the excitons gives rise to a sequence of photons, each exhibiting a different energy. The photon produced by the recombination of the final exciton can be spectrally selected, hence, giving exactly one photon for each excitation event, independent of the number of excitons initially excited. ${ }^{1}$ Semiconductor QDs therefore provide highly compact and efficient on-demand sources of single photons.

While GaAs-based QD single photon sources at 900-1300 $\mathrm{nm}^{2-4}$ and InP-based sources in the telecoms C-band at 1550 $\mathrm{nm}^{5}$ have been demonstrated, a Si-based C-band source, allowing direct integration with Si-based drive electronics, is highly desirable. Extension of the emission wavelength from QDs grown within a GaAs matrix has been demonstrated by capping InAs QDs with a thin GaAsSb layer; room temperature emission out to $\sim 1600 \mathrm{~nm}$ has been achieved. ${ }^{6-23}$ Detailed structural studies reveal that capping InAs QDs with GaAsSb prevents the decomposition observed when capping with GaAs. $\mathrm{GaAsSb}$ capped QDs were found to have a height approximately twice that of GaAs capped dots; the lateral sizes appeared to be very similar. The initial height of the QDs was found to be retained for a $\mathrm{Sb}$ composition greater than $14 \%{ }^{22}$ This suppression of the decomposition was attributed to the reduced lattice mismatch between InAs and GaAsSb, compared to InAs and GaAs. ${ }^{11}$ In addition, it is well documented that capping QDs with a strain reducing layer (for example, InGaAs, but also GaAsSb) results in a red shift of the emission. ${ }^{23}$ A third mechanism contributing to the extension of the emission wavelength is a transition to a type-II system for $\mathrm{Sb}$ compositions above $\sim 14 \%$, ${ }^{6,8,13,17,23}$ with the hole localized in the GaAsSb two-dimensional layer and the electron in the QD. Related to the structures studied in the present work are GaSb QDs and quantum rings grown in a GaAs matrix. ${ }^{24-31}$ These also form a type-II system but with the electrons localized in the GaAs and holes in the QD or ring. ${ }^{25,29}$ Emission wavelengths around $\sim 1.3 \mu \mathrm{m}$ are typically observed. $^{24,25,27}$

In this paper we report the observation of single QD emission at wavelengths as long as $1540 \mathrm{~nm}$ from InAs QDs capped with a $6 \mathrm{~nm} \mathrm{GaAs}{ }_{0.74} \mathrm{Sb}_{0.26}$ layer and grown directly on a $\mathrm{Si}$ substrate. As the number of QDs probed decreases, the inhomogeneously broadened emission breaks up into a small number of sharp lines, each line corresponding to emission from excitons localized in different QDs. Comparison is made

Received: March 22, 2017

Published: June 20, 2017 
to a nominally identical reference sample grown on a GaAs substrate. The present results suggest the potential for Si-based single photon sources emitting in the C-band.

Two samples were grown by solid-source III-V molecular beam epitaxy (MBE) on Si and [001] GaAs substrates. Both active regions were identical and consisted of $50 \mathrm{~nm}$ of GaAs followed by a single low density InAs QD layer capped with 6 $\mathrm{nm}$ of $\mathrm{GaAs}_{0.74} \mathrm{Sb}_{0.26}$ and $50 \mathrm{~nm}$ of GaAs. These layers were grown at $\sim 510{ }^{\circ} \mathrm{C}$. Both sides were clad with a $100 \mathrm{~nm}$ $\mathrm{Al}_{0.4} \mathrm{Ga}_{0.6} \mathrm{As}$ layer grown at $610{ }^{\circ} \mathrm{C}$ and the structure was terminated with a $50 \mathrm{~nm} \mathrm{GaAs}$ layer grown at $580{ }^{\circ} \mathrm{C}$. The $\mathrm{Si}$ structure was grown directly on an n-doped $\mathrm{Si}(100)$ substrate with $4^{\circ}$ offcut to the [011] plane. This substrate orientation is used to suppress the formation of antiphase domains in the III-V material. ${ }^{32}$ Oxide desorption of the substrate was performed at $900{ }^{\circ} \mathrm{C}$ for $15 \mathrm{~min}$, followed by a cool down to $370{ }^{\circ} \mathrm{C}$ and the growth of a $200 \mathrm{~nm}$ GaAs nucleation layer comprising an optimized two-step growth scheme. ${ }^{33-35}$ An 800 $\mathrm{nm}$ GaAs buffer layer was deposited, followed by three dislocation filter layers (DFLs). These were included to reduce the number of dislocations, which form at the Si:III-V interface, propagating into the device active region where they degrade the optical efficiency. DFLs are highly effective in improving the performance of $\mathrm{QD}$ lasers grown on $\mathrm{Si}$ substrates, reducing the dislocation density from $\sim 10^{9} \mathrm{~cm}^{-2}$ at the Si:III-V interface to $\sim 10^{6} \mathrm{~cm}^{-2}$ in the active region. ${ }^{36}$ The Abstract graphic shows a schematic of the Si-substrate structure, including the two methods used to isolate a small number of QDs: etched micropillars and holes in a metal mask. The vertical scale is exaggerated to clearly show the different epitaxial layers; in the actual structures the aspect ratio of the pillars is close to unity (see Figure 3 below).

Figure 1a shows a dark field 002 transmission electron microscopy (TEM) image of a single GaAsSb capped InAs QD. A number of QDs were examined and found to be of similar size and shape; the image shown is a representative example. Tilting of the TEM specimen showed that it was $\sim 50 \mathrm{~nm}$ thick, completely containing the QD; the specimen shape and size revealed in this image should therefore be accurate. The contrast is roughly proportional to the square of the difference in mean atomic number of the group III and V atoms $\left(\bar{Z}_{\mathrm{III}}-\right.$ $\left.\bar{Z}_{\mathrm{V}}\right)^{2}$, with an amplitude integrated through the specimen thickness; ${ }^{37}$ thus, the GaAsSb layer appears bright, while the GaAs matrix is dark. The InAs QD also appears dark due to a change in sign of $\left(\bar{Z}_{\text {III }}-\bar{Z}_{\mathrm{V}}\right)$ compared to the GaAsSb cap. The InAs $Q D$ has a height $\sim 8 \mathrm{~nm}$ and width $\sim 23 \mathrm{~nm}$. These values are reasonably consistent with previous cross-sectional scanning tunneling microscopy (X-STM) studies of a very similar structure, which found a height of $9.5 \mathrm{~nm}$ and a width of $32 \mathrm{~nm}$, although the QDs had a more pyramidal shape. ${ }^{11}$ The GaAsSb capping layer appears to be nonuniform close to the QD, with a "ring-like" variation in thickness. A similar structure was observed in X-STM ${ }^{11}$ for samples where the GaAsSb was grown at $500{ }^{\circ} \mathrm{C}$. This was attributed to migration of material away from the apex of the dot, hence minimizing the strain.

Figure $1 \mathrm{~b}$ shows an annular dark field (ADF) scanning TEM image of a second GaAsSb capped InAs QD along with energydispersive X-ray spectroscopy (EDX) composition maps of the elements Ga, As, In and Sb. Contrast in the ADF-STEM image increases with atomic number, and the $\mathrm{QD}$ appears only slightly brighter than the surrounding material. The $\mathrm{QD} / \mathrm{cap}$ interface in the In map is diffuse, making it difficult to accurately determine the QD height; the In composition profile
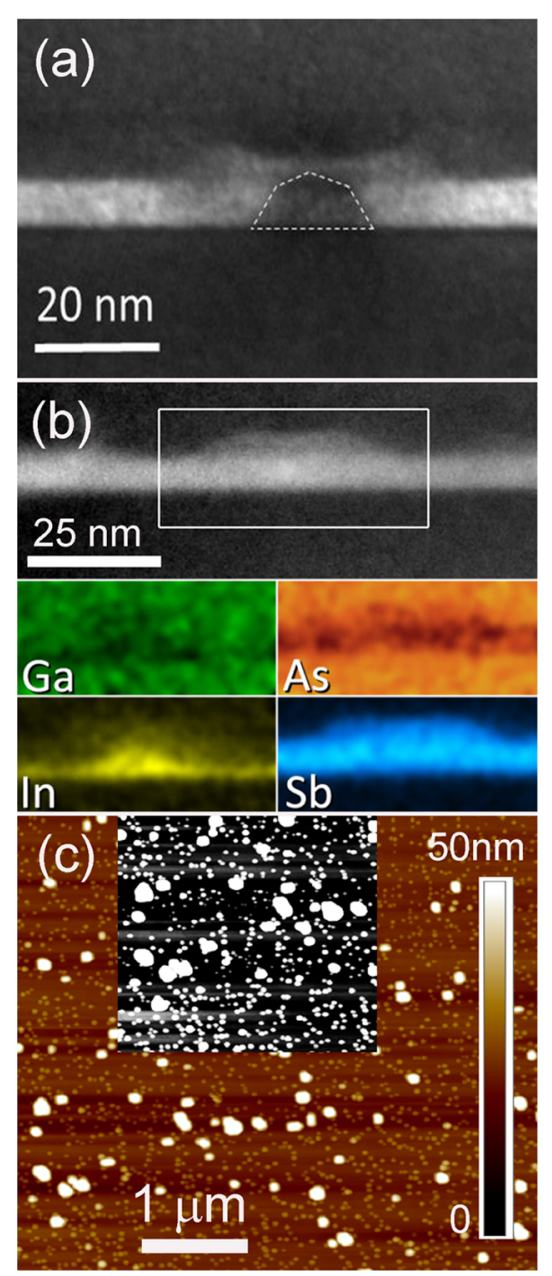

Figure 1. (a) High resolution dark field TEM image of a single GaAsSb-capped InAs QD (a dotted line demarcates the QD from the surrounding material). The bright region is the GaAsSb. (b) Annular dark field STEM image of a single GaAsSb-capped InAs QD, with energy-dispersive $\mathrm{X}$-ray composition analysis. The white box shows the region selected for the compositional analysis. (c) AFM image of uncapped QDs from a calibration sample showing the two QD subsets. The smaller subset of QDs is more clearly seen in the black and white inset with enhanced contrast.

indicates a value between 6.5 and $9.5 \mathrm{~nm}$. Away from the QD, the GaAsSb layer has a thickness of $\sim 5-6 \mathrm{~nm}$, consistent with a value of $6 \mathrm{~nm}$ estimated from the growth conditions. The In map reveals a nonzero In composition in the nominal GaAsSb capping layer, a result of In diffusion or segregation during growth. The incorporation of In will reduce the band gap of this layer.

Figure 1c shows an atomic force microscopy (AFM) image of an uncapped layer of QDs grown under the same conditions as the capped QDs used for optical studies. Visible in the image are two subsets of QDs. The distribution of the large QDs exhibits a significant spatial variation with a low average density, estimated to be of order $5 \mu \mathrm{m}^{-2}$. These dots also exhibit a broad size distribution. The subset of smaller QDs has a much larger density $\sim 80 \mu \mathrm{m}^{-2}$. We show below that the larger QDs are responsible for emission in the $1500 \mathrm{~nm}$ spectral region.

The structural images shown in Figure 1a,b were obtained for a sample grown on a GaAs substrate, the AFM image is for growth on Si. Previous structural studies of QDs grown on both $\mathrm{Si}$ and GaAs substrates have shown no significant differences in 
shape, size, and density. This is expected as a large thickness $(>1 \mu \mathrm{m})$ of GaAs is deposited on the Si substrate before the QDs are grown. In addition, optical studies have revealed the QDs to have very similar properties apart from a small energy shift of the emission for QDs grown on a Si substrate. ${ }^{36}$ This shift is attributed to residue strain $(0.24 \%$ at a temperature of $77 \mathrm{~K}$ ) that results from the different $\mathrm{III}-\mathrm{V}$ and $\mathrm{Si}$ thermal expansion coefficients and cool down following growth. ${ }^{36}$ In the optical data present below, both sets of QDs emit at essentially the same wavelengths for the $\mathrm{Si}$ and GaAs substrate samples, confirming very similar physical structures.

Initial optical characterization was performed for a sample temperature of $77 \mathrm{~K}$ with photoluminescence (PL) excited by a $638 \mathrm{~nm}$ laser focused to a diameter $\sim 200 \mu \mathrm{m}$; probing approximately 6000 of the larger dots. Figure 2a shows spectra
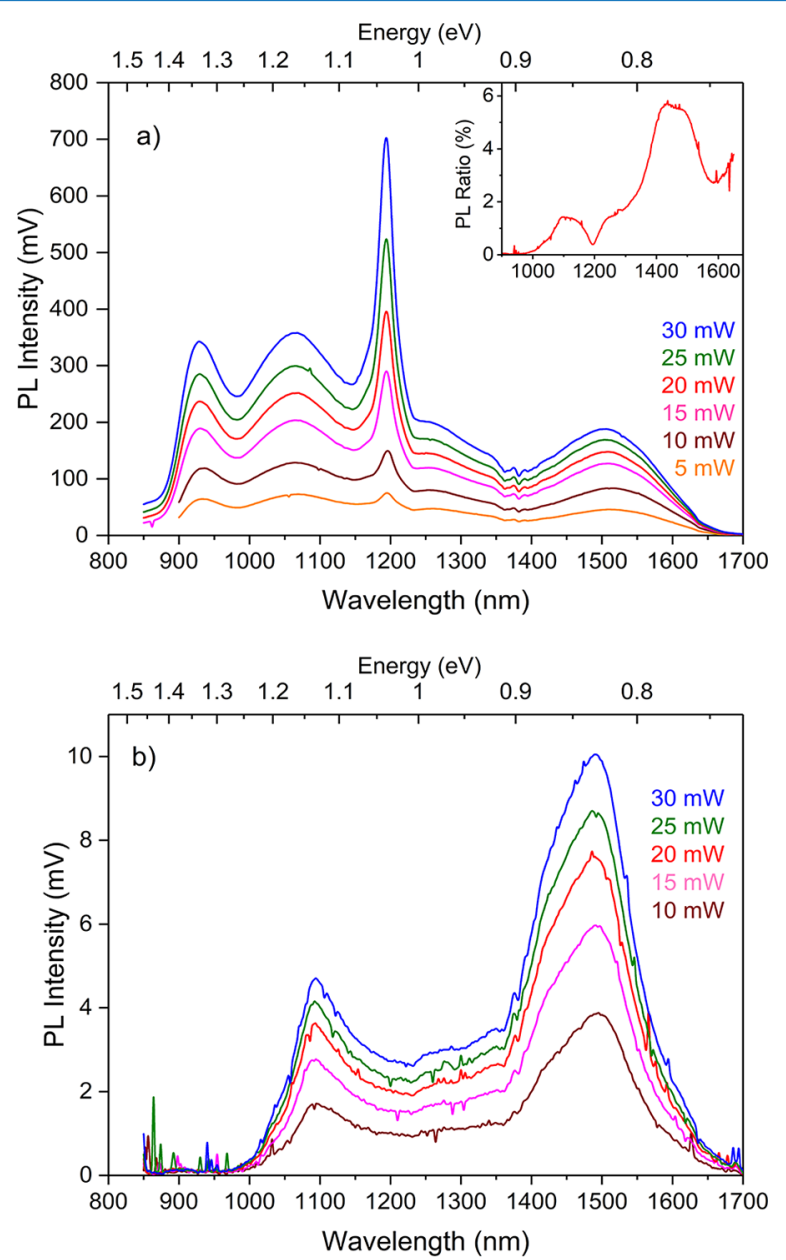

Figure 2. Macro PL (77 K) spectra: (a) Sample grown on a GaAs substrate; (b) Sample grown on a Si substrate. A sharp feature at $\sim 1270 \mathrm{~nm}$ in (b), produced by second order scattered laser light, has been removed to aid clarity. The inset to (a) shows the ratio of the PL intensity for the $\mathrm{Si}$ to GaAs grown samples for a laser power of 30 $\mathrm{mW}$.

recorded for excitation powers between 5 and $30 \mathrm{~mW}$ (三 20-100 $\mathrm{Wcm}^{-2}$ ) for the GaAs substrate sample. For the Si substrate sample (Figure $2 \mathrm{~b}$ ) the power range was limited to 10-30 $\mathrm{mW}$ because of weaker PL. Both samples exhibit a broad $(110 \pm 5 \mathrm{meV}$ and $80 \pm 2 \mathrm{meV}$ for the GaAs and $\mathrm{Si}$ substrate samples respectively) long wavelength feature centered at $\sim 1500 \mathrm{~nm}$, but with emission extending beyond
$1650 \mathrm{~nm}$. This emission is attributed to the subset of larger QDs, the large variation in dot size observed in AFM (Figure 1c) resulting in significant inhomogeneous spectral broadening. The cooled Ge detector used to measure the PL falls off rapidly in sensitivity above $\sim 1400 \mathrm{~nm}$ and cuts off fully at $\sim 1700 \mathrm{~nm}$. An approximate correction for this spectral response suggests the presence of strong emission to at least $1650 \mathrm{~nm}$. Other features present in Figure $2 \mathrm{a}$ are emission from the InAs wetting layer at $\sim 920 \mathrm{~nm}$, the subset of smaller QDs at 1070 $\mathrm{nm}$ and the GaAsSb capping layer at $\sim 1200 \mathrm{~nm}$. The estimated $\mathrm{GaAsSb}$ band gap for $26 \% \mathrm{Sb}$ is $1204 \mathrm{~nm}$, very close to the measured value. ${ }^{38}$ The emission from the subset of smaller QDs is brighter than that of the larger QDs, in agreement with their relative densities (Figure 1c). Previous studies of similar structures show only the long wavelength emission, 7,8 indicating a single set of QDs. Structural studies of these samples $^{11}$ reveal QD parameters very similar to those determined from the present TEM and STEM measurements, indicating that the dots shown in Figure 1 give rise to the long wavelength emission at $\sim 1500 \mathrm{~nm}$.

The long wavelength $\mathrm{QD}$ emission from the Si substrate sample (Figure $2 \mathrm{~b}$ ) is $\sim 20$ weaker in comparison to the GaAs sample. This is attributed to dislocations reaching the active region where they act as nonradiative centers. These have a much larger effect on emission from the InAs wetting and $\mathrm{GaAsSb}$ capping layers as captured carriers are able to migrate in-plane until they reach a dislocation. No emission is observed from either layer in the $\mathrm{Si}$ substrate sample. In contrast, although the QD emission is reduced in intensity, it is still visible because carrier capture by a $Q D$ inhibits subsequent migration to a dislocation. ${ }^{39}$ In contrast to the GaAs substrate sample, emission from the lower density, larger QDs is more intense than that of the higher density, smaller QDs. This most likely reflects the much deeper confining potential of the larger QDs, which provides a strong barrier preventing carrier migration to dislocations. The differing behavior of the $\mathrm{QD}$ and bulk/2D layers is demonstrated in the inset to Figure 2a, which plots the PL intensity ratio of the $\mathrm{Si} / \mathrm{GaAs}$ samples for a laser power of $30 \mathrm{~mW}$. Although the ratio is always less than unity, peaks are observed at $\sim 1100$ and $\sim 1500 \mathrm{~nm}$, corresponding to emission from the two subsets of QDs, demonstrating their reduced sensitivity to nonradiative defects. The optical efficiency of the Si substrate sample could be increased by including annealing steps during growth to further improve the effectiveness of the DFLs; this has been shown to significantly increase the optical efficiency of test structures and the performance of QD lasers. ${ }^{36}$

In order to observe single QD emission, the samples were etched into arrays of $1 \mu \mathrm{m}$ diameter pillars with a pitch of $5 \mu \mathrm{m}$ (Figure 3). The arrays were patterned using e-beam lithography and etched to a depth $\sim 450 \mathrm{~nm}$ using inductive coupled plasma etching (ICP). The samples were cooled to $\sim 20 \mathrm{~K}$ in a closed loop helium cryostat. A 50× IR high NA microscope objective lens was used for both excitation and PL collection, giving a laser spot diameter of $\sim 3 \mu \mathrm{m}$ for excitation with a $532 \mathrm{~nm}$ laser. PL was detected by a thermoelectric cooled InGaAs array. Data from the InGaAs array was taken at $-90{ }^{\circ} \mathrm{C}$ to reduce thermal noise/dark currents, giving a quantum efficiency of $\sim 76 \%$ at $1550 \mathrm{~nm}$.

Figure 4 shows typical low power spectra from four pillars: (a) GaAs substrate, laser power $0.2 \mu \mathrm{W}$, and (b) Si substrate, laser power $2 \mu \mathrm{W}$. All spectra show a number of sharp lines on a broad background. Each sharp line is attributed to emission 


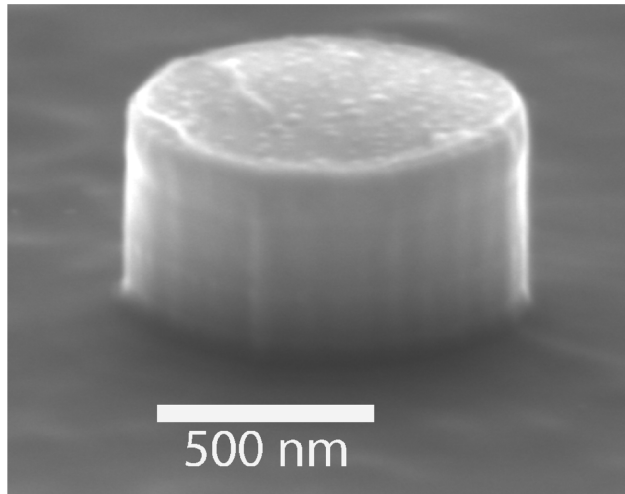

Figure 3. Scanning electron microscope image of a $1 \mu \mathrm{m}$ diameter etched pillar on a GaAs substrate.

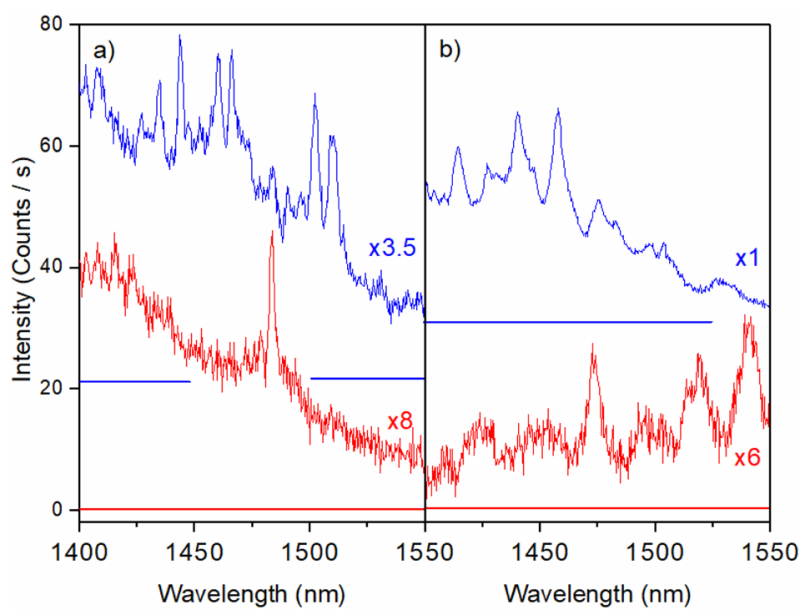

Figure 4. PL spectra $(20 \mathrm{~K})$ for different micropillars: (a) GaAs substrate excited with $0.2 \mu \mathrm{W}$; (b) Si substrate excited with $2 \mu \mathrm{W}$. The spectra have been offset vertically for clarity. The solid horizontal colored lines show the zero levels for the relevant spectra.

from one of the small number of QDs isolated by the $\sim 1 \mu \mathrm{m}$ diameter pillars. For the $\mathrm{Si}$ substrate sample, the longest observed single dot emission is $1540 \mathrm{~nm}$, for the GaAs substrate sample it is $1510 \mathrm{~nm}$. To the best of our knowledge this is the first reported observation of single $\mathrm{QD}$ emission at $1500 \mathrm{~nm}$ from QDs grown on Si or GaAs substrates. Si-based single $\mathrm{QD}$ emission at this wavelength is particularly significant as it provides the potential for single photon sources emitting at the minimum absorption of silica optical fibers and with simpler integration with drive electronics.

A greater variation in the PL intensity between different pillars fabricated from the Si substrate sample is observed than is the case for the GaAs sample. This stronger variation may reflect the presence of a random number of dislocations in different pillars. However, the incorporation of the three DFLs should reduce the defect density in the active region to $\sim 10^{6}$ $\mathrm{cm}^{-2}$ corresponding to an average of only one defect for every hundred pillars.

For both the GaAs and Si substrate samples the single QD emission sits on a broad background (Figure 4). For the laser powers used to record these spectra $(0.2$ and $2 \mu \mathrm{W}$ for the GaAs and $\mathrm{Si}$ samples, respectively), the QD peak to background intensity ratio is approximately unity; for higher powers (above $2 \mu \mathrm{W}$ for the GaAs sample), the ratio decreases, reaching a value $\sim 0.1$ for a power of $20 \mu \mathrm{W}$ (GaAs sample).
The origin of this background emission is unclear but is most likely the result of additional exciton confining centers in close proximity to the QD. For a single photon source the presence of a background would degrade the fidelity via the production of additional photons at the same wavelength as the QD emission. The current samples were designed to study the properties of the QDs and were not optimized for single photon sources. Further studies are necessary to reduce the background emission, for example, by optimizing confinement of carriers close to the QD and lowering the QD density. In addition, the collected signal from the QD's emission can be dramatically increased by the addition of an optical cavity. ${ }^{40}$

Figure 5 shows spectra for an excitation range of 0.4 to 300 $\mu \mathrm{W}\left(\equiv 6-4500 \mathrm{Wcm}^{-2}\right)$ for a pillar fabricated from the GaAs

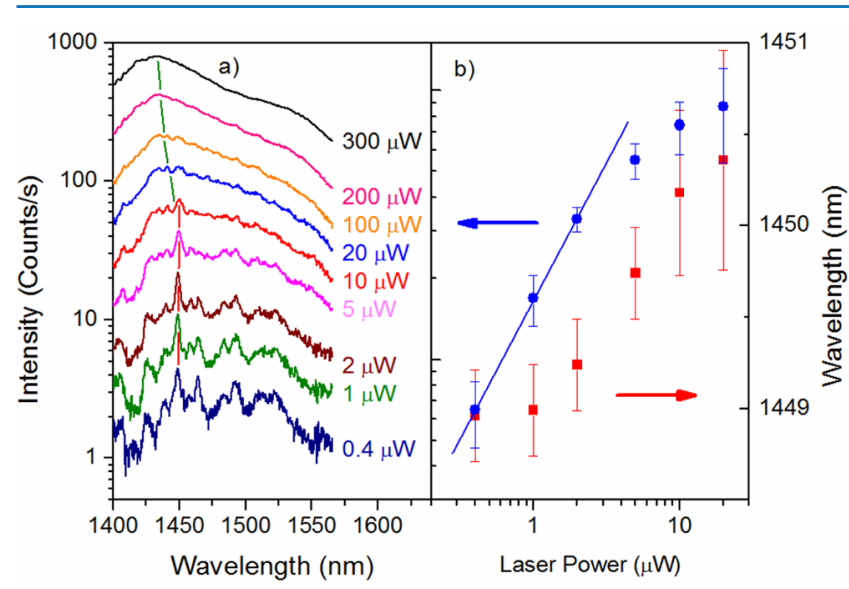

Figure 5. (a) PL spectra $(20 \mathrm{~K})$ of a $1 \mu \mathrm{m}$ micropillar on a GaAs substrate for an excitation power range $0.4-300 \mu \mathrm{W}$. The green (red) lines are guides to the eye and show the positions of the continuum maximum (sharp feature at $\sim 1450 \mathrm{~nm}$ ). (b) Plots of the wavelength (red) and total intensity (blue) against incident laser power for the sharp line at $\sim 1450 \mathrm{~nm}$.

substrate sample. This sample was chosen for detailed study as the higher PL efficiency allowed for a greater range of laser powers. At high excitation powers a continuous spectrum is observed, with a maximum at $1430 \mathrm{~nm}$ and emission extending beyond $1560 \mathrm{~nm}$. As the power is decreased, the emission peak shifts to longer wavelength before breaking up into a number of discrete peaks, the positions of which shift very weakly with excitation power. This behavior is similar to that reported for type-II GaSb/GaAs quantum rings. ${ }^{41}$ Here the blue shift of the emission was attributed to emission from higher energy QD states rather than the band bending predicted for a type-II system. ${ }^{9}$ The right-hand panel of Figure 5 shows the wavelength and total intensity (obtained by multiplying the line width by the peak intensity) of the sharp feature observed close to $1450 \mathrm{~nm}$, plotted against laser power. At low powers the intensity exhibits a nearly linearly dependence on laser power (gradient of $0.95 \pm 0.06$ on a log-log plot) before saturating at high powers. This is consistent with single-exciton recombination. No evidence for additional lines at high power, representing multiexciton recombination, is observed; these may be obscured by the broad background. The $1450 \mathrm{~nm}$ feature exhibits a weak redshift at high powers which may result from sample heating. There is also an increase in line width from $3.6 \mathrm{~nm}$ at $0.4 \mu \mathrm{W}$ to $6 \mathrm{~nm}$ at $10 \mu \mathrm{W}(\equiv 2.3-3.8 \mathrm{meV})$. A similar power dependent behavior to that shown in Figure 5 is observed for the sample grown on a $\mathrm{Si}$ substrate. The PL 
intensity exhibits an initial approximate linear intensity increase, with the rate decreasing at higher powers. For this sample the saturation at high powers is less pronounced because powers higher than obtainable with the current experimental setup are required, given the lower optical efficiency. The maximum single QD emission intensity is comparable for the two samples.

The line widths of the single QD emission from both the $\mathrm{Si}$ and GaAs substrate samples show a range of values between $\sim 1.3$ and $5 \mathrm{meV}$. These values are significantly larger than observed in type-I QD systems where line widths of $\sim 10 \mu \mathrm{eV}$ can be typically achieved for high quality InAs dots grown in a GaAs matrix and for nonresonant optical excitation. ${ }^{42}$ The current experimental system has measured line widths as small as $200 \mu \mathrm{eV}$ for type-II GaSb/GaAs quantum rings emitting at $1200 \mathrm{~nm}$, indicating that the current line widths are not resolution limited. ${ }^{41}$ It is known that fluctuating carrier occupancy of states in the vicinity of a $Q D$ can increase the emission line width via local electric fields and the resultant quantum confined Stark effect (QCSE). ${ }^{43,44}$ The QCSE for an infinite depth quantum well is proportional to the fourth power of the well width. ${ }^{45}$ Hence, in a type-II system, with spatially separated electrons and holes, the response to fluctuating electric fields is likely to be much larger than in a type-I system where the spatial separation of the electron and hole is significantly smaller. Calculations for InAs QDs capped with a 6 $\mathrm{nm}$ GaAsSb layer give large zero-field dipole moments of 2.3 and $9 \mathrm{~nm}$ along the growth axis and in-plane, respectively. ${ }^{21} \mathrm{In}$ contrast, a much smaller growth axis value of $0.4 \mathrm{~nm}$ has been measured for type-I InAs QDs within a GaAs matrix. ${ }^{46}$ Similarly type-I QDs with axial symmetry should possess a zero or, at most, small in-plane dipole moment.

Line width broadening by charge fluctuations can be particularly significant in etched mesa structures where there may be a large number of surface states close to the QDs. To study possible effects of side wall surface states, a small number of quantum dots were isolated by forming $1 \mu \mathrm{m}$ apertures in an opaque metal mask. Such structures avoid the presence of etched semiconductor surfaces. Again both samples exhibit a series of sharp lines, with line widths in the range $\sim 3-5 \mathrm{meV}$. These slightly larger line widths may result from the need to use significant higher laser powers $(5 \mu \mathrm{W}$ and $20 \mu \mathrm{W}$ for the GaAs and $\mathrm{Si}$ substrate structures, respectively) due to the reduced in/out coupling efficiency of the apertures; measurements discussed above show a power dependent emission line width. There is, hence, no evidence for significantly lower emission line widths in the aperture structures, demonstrating that surface state induced fluctuating electric fields are not the dominant cause of the observed $\sim 1 \mathrm{meV}$ values. However, it is not possible to rule out the effects of charge fluctuations in bulk states which may have a relatively large effect due to the high laser powers and the type-II nature of the optical transitions.

A possible additional contribution to the $\mathrm{QD}$ line width results from the localization of the hole in the GaAsSb layer. Thickness fluctuations result in a broadening of the emission, with further broadening due to alloy fluctuations. ${ }^{47}$ It is difficult to estimate the size of this broadening; the TEM image of Figure 1a shows a highly nonuniform GaAsSb layer thickness and X-STM studies of similar samples reveal a nonuniform $\mathrm{Sb}$ composition. ${ }^{11,22}$ A structural study of InAs QDs capped with a $\mathrm{GaAs}_{0.78} \mathrm{Sb}_{0.22}$ layer shows significant alloy fluctuations $(\sim 12 \%)$ with $\mathrm{Sb}$-rich clusters of lateral dimensions between 10 and 20 $\mathrm{nm} .^{22}$ For relatively large composition and/or layer thickness fluctuations, the GaAsSb layer will break up into a series of quasi-QDs ${ }^{48}$ with full spatial localization of the hole. However, in this case, spectral broadening is still possible via the population of different hole states during the many recombination processes that occur within the integration time required to acquire the PL spectra.

In conclusion, we have reported the observation of single QD emission at telecoms wavelengths from QDs grown on a Si substrate. Emission at $1500 \mathrm{~nm}$ is achieved by capping InAs QDs with a thin GaAsSb layer which results in larger dots and a type-II system. Line widths are significantly larger than observed for type-I InAs QDs grown in a GaAs matrix. The broadening observed in the current samples is attributed to the high carrier densities required to excite the PL, with a possible contribution from alloy and layer thickness fluctuations in the hole confining GaAsSb layer. The observation of single QD emission at $1500 \mathrm{~nm}$ gives the potential for single photon sources at telecommunication wavelengths. With further growth developments the current structure could be made compatible with CMOS technology, giving the potential for direct integration with $\mathrm{Si}$ drive electronics.

\section{ASSOCIATED CONTENT}

\section{Supporting Information}

The Supporting Information is available free of charge on the ACS Publications website at DOI: 10.1021/acsphotonics.7b00276.

Example of PL corrected for Ge detector response and example spectra from $1 \mu \mathrm{m}$ apertures in an opaque metal mask (PDF).

\section{AUTHOR INFORMATION}

\section{Corresponding Author}

*E-mail: d.mowbray@sheffield.ac.uk.

ORCID $\odot$

Jiang Wu: 0000-0003-0679-6196

Robert J. Young: 0000-0002-5719-2205

David J. Mowbray: 0000-0002-7673-6837

Notes

The authors declare no competing financial interest.

\section{ACKNOWLEDGMENTS}

The authors would like to acknowledge the support of the Engineering and Physical Sciences Research Council (EPSRC), Grant No. EP/J012882/1 (Sheffield), EP/K50421X/1 and EP/ L01548X/1 (Lancaster), EP/J012904/1 (UCL), and EP/ J013048/1 (Warwick). This work was supported by the Royal Society through a University Research Fellowship (UF110555) held by R.J.Y. This material is based on work supported by the Air Force Office of Scientific Research under Award Number FA9550-16-1-0276.

\section{REFERENCES}

(1) Shields, A. J. Semiconductor quantum light sources. Nat. Photonics 2007, 1, 215.

(2) Michler, P.; Kiraz, A.; Becher, C.; Schoenfeld, W. V.; Petroff, P. M.; Zhang, L.; Hu, E.; Imamoglu, A. A Quantum dot-single photon turnstile device. Science 2000, 290, 2282.

(3) Zinoni, C.; Alloing, B.; Monat, C.; Zwiller, V.; Li, L. H.; Fiore, A.; Lunghi, L.; Gerardino, A.; de Riedmatten, H.; Zbinden, H.; Gisin, N. Time-resolved and antibunching experiments on single quantum dots at 1300nm. Appl. Phys. Lett. 2006, 88, 131102. 
(4) Ward, M. B.; Farrow, T.; See, P.; Yuan, Z. L.; Karimov, O. Z.; Bennett, A. J.; Shields, A. J.; Atkinson, P.; Cooper, K.; Ritchie, D. A. Electrically driven telecommunication wavelength single-photon source. Appl. Phys. Lett. 2007, 90, 063512.

(5) Miyazawa, T.; Takemoto, K.; Sakuma, Y.; Hirose, S.; Usuki, T.; Yokoyama, N.; Takatsu, M.; Arakawa, Y. Single-Photon Generation in the $1.55-\mu \mathrm{m}$ Optical-Fiber Band from an InAs/InP Quantum Dot. Jpn. J. Appl. Phys. 2005, 44, L620.

(6) Ripalda, J. M.; Granados, D.; Gonzalez, Y.; Sanchez, A. M.; Molina, S. I.; Garcia, J. M. Room temperature emission at $1.6 \mu \mathrm{m}$ from InGaAs quantum dots capped with GaAsSb. Appl. Phys. Lett. 2005, 87, 202108.

(7) Liu, H. Y.; Steer, M. J.; Badcock, T. J.; Mowbray, D. J.; Skolnick, M. S.; Navaretti, P.; Groom, K. M.; Hopkinson, M.; Hogg, R. A. Longwavelength light emission and lasing from InAs/GaAs quantum dots covered by a GaAsSb strain-reducing layer. Appl. Phys. Lett. 2005, 86, 143108.

(8) Liu, H. Y.; Steer, M. J.; Badcock, T. J.; Mowbray, D. J.; Skolnick, M. S.; Suarez, F.; Ng, J. S.; Hopkinson, M.; David, J. P. R. Roomtemperature $1.6 \mu \mathrm{m}$ light emission from InAs/GaAs quantum dots with a thin GaAsSb cap layer. J. Appl. Phys. 2006, 99, 046104.

(9) Jin, C. Y.; Liu, H. Y.; Zhang, S. Y.; Jiang, Q.; Liew, S. L.; Hopkinson, M.; Badcock, T. J.; Nabavi, E.; Mowbray, D. J. Optical transitions in type-II InAs/GaAs quantum dots covered by a GaAsSb strain-reducing layer. Appl. Phys. Lett. 2007, 91, 021102.

(10) Jang, Y. D.; Badcock, T. J.; Mowbray, D. J.; Skolnick, M. S.; Park, J.; Lee, D.; Liu, H. Y.; Steer, M. J.; Hopkinson, M. Carrier lifetimes in type-II InAs quantum dots capped with a GaAsSb strain reducing layer. Appl. Phys. Lett. 2008, 92, 251905.

(11) Ulloa, J. M.; Drouzas, I. W. D.; Koenraad, P. M.; Mowbray, D. J.; Steer, M. J.; Liu, H. Y.; Hopkinson, M. Suppression of InAs/GaAs quantum dot decomposition by the incorporation of a GaAsSb capping layer. Appl. Phys. Lett. 2007, 90, 213105.

(12) Akahane, K.; Yamamoto, N.; Ohtani, N. Long-wavelength light emission from InAs quantum dots covered by GaAsSb grown on GaAs substrates. Phys. E 2004, 21, 295.

(13) Ulloa, J. M.; Llorens, J. M.; del Moral, M.; Bozkurt, M.; Koenraad, P. M.; Hierro, A. Analysis of the modified optical properties and band structure of $\mathrm{GaAs}_{1-\mathrm{x}} \mathrm{Sb}_{\mathrm{x}}$-capped InAs/GaAs quantum dots. J. Appl. Phys. 2012, 112, 074311.

(14) Haxha, V.; Drouzas, I.; Ulloa, J. M.; Bozkurt, M.; Koenraad, P. M.; Mowbray, D. J.; Liu, H. Y.; Steer, M. J.; Hopkinson, M.; Migliorato, M. A. Role of segregation in InAs/GaAs quantum dot structures capped with a GaAsSb strain-reduction layer. Phys. Rev. B: Condens. Matter Mater. Phys. 2009, 80, 165334.

(15) Zíková, M.; Hospodková, A.; Pangrác, J.; Oswald, J.; Krčil, P.; Hulicius, E.; Komninou, P.; Kioseoglou, J. MOVPE prepared InAs/ $\mathrm{GaAs}$ quantum dots covered by GaAsSb layer with long wavelength emission at $1.8 \mu \mathrm{m}$. J. Cryst. Growth 2015, 414, 167.

(16) Klenovský, P.; Křápek, V.; Munzar, D.; Humlíček, J. Electronic structure of InAs quantum dots with GaAsSb strain reducing layer: Localization of holes and its effect on the optical properties. Appl. Phys. Lett. 2010, 97, 203107.

(17) Hsu, W.-T.; Liao, Y.-A.; Hsu, F.-C.; Chiu, P.-C.; Chyi, J.-I.; Chang, W.-H. Effects of GaAsSb capping layer thickness on the optical properties of InAs quantum dots. Appl. Phys. Lett. 2011, 99, 073108.

(18) Pavarelli, N.; Ochalski, T. J.; Liu, H. Y.; Gradkowski, K.; Schmidt, M.; Williams, D. P.; Mowbray, D. J.; Huyet, G. Competitive carrier interactions influencing the emission dynamics of GaAsSbcapped InAs quantum dots. Appl. Phys. Lett. 2012, 101, 231109.

(19) Liu, H. Y.; Steer, M. J.; Badcock, T. J.; Mowbray, D. J.; Skolnick, M. S.; Navaretti, P.; Groom, K. M.; Hopkinson, M.; Hogg, R. A. Longwavelength light emission and lasing from quantum dots covered by a GaAsSb strain-reducing layer. Appl. Phys. Lett. 2005, 86, 143108.

(20) Utrilla, A. D.; Ulloa, J. M.; Guzman, A.; Hierro, A. Impact of the $\mathrm{Sb}$ content on the performance of GaAsSb-capped InAs/GaAs quantum dot lasers. Appl. Phys. Lett. 2013, 103, 111114.

(21) Llorens, J. M.; Wewior, L.; Cardozo de Oliveira, E. R.; Ulloa, J. M.; Utrilla, A. D.; Guzman, A.; Hierro, A.; Alen, B. Type II InAs/
GaAsSb quantum dots: Highly tunable exciton geometry and topology. Appl. Phys. Lett. 2015, 107, 183101.

(22) Ulloa, J. M.; Gargallo-Caballero, R.; Bozkurt, M.; del Moral, M.; Guzmán, A.; Koenraad, P. M.; Hierro, A. GaAsSb-capped InAs quantum dots: From enlarged quantum dot height to alloy fluctuations. Phys. Rev. B: Condens. Matter Mater. Phys. 2010, 81, 165305.

(23) Zíková, M.; Hospodková, A.; Pangrác, J.; Oswald, J.; Hulicius, E. Comparison of MOVPE grown GaAs, InGaAs and GaAsSb covering layers for different InAs/GaAs quantum dot applications. J. Cryst. Growth 2017, 464, 59.

(24) Kamarudin, M. A.; Hayne, H.; Young, R. J.; Zhuang, Q. D.; Ben, T.; Molina, S. I. Tuning the properties of exciton complexes in selfassembled $\mathrm{GaSb} / \mathrm{GaAs}$ quantum rings. Phys. Rev. B: Condens. Matter Mater. Phys. 2011, 83, 115311.

(25) Carrington, P. J.; Young, R. J.; Hodgson, P. D.; Sanchez, A. M.; Hayne, M.; Krier, A. Long-Wavelength Photoluminescence from Stacked Layers of High Quality Type-II GaSb/GaAs Quantum Rings. Cryst. Growth Des. 2013, 13, 1226.

(26) Gradkowski, K.; Pavarelli, N.; Ochalski, T. J.; Williams, D. P.; Tatebayashi, J.; Huyet, G.; O’Reilly, E. P.; Huffaker, D. L. Complex emission dynamics of type-II GaSb/GaAs quantum dots. Appl. Phys. Lett. 2009, 95, 061102.

(27) Richter, J.; Strassner, J.; Loeber, T. H.; Fouckhardt, H.; Nowozin, T.; Bonato, L.; Bimberg, D.; Braam, D.; Lorke, A. GaSb quantum dots on GaAs with high localization energy of $710 \mathrm{meV}$ and an emission wavelength of $1.3 \mu \mathrm{m}$. J. Cryst. Growth 2014, 404, 48.

(28) Hogg, R. A.; Suzuki, K.; Tachibana, K.; Finger, L.; Hirakawa, K.; Arakawa, Y. Optical spectroscopy of self-assembled type II GaSb/GaAs quantum dot structures grown by molecular beam epitaxy. Appl. Phys. Lett. 1998, 72, 2856.

(29) Gradkowski, K.; Pavarelli, N.; Ochalski, T. J.; Williams, D. P.; Tatebayashi, J.; Huyet, G.; O’Reilly, E. P.; Huffaker, D. L. Complex emission dynamics of type-II GaSb/GaAs quantum dots. Appl. Phys. Lett. 2009, 95, 061102.

(30) Timm, R.; Eisele, H.; Lenz, A.; Ivanova, L.; Balakrishnan, G.; Huffaker, D. L.; Dähne, M. Self-Organized Formation of GaSb/GaAs Quantum Rings. Phys. Rev. Lett. 2008, 101, 256101.

(31) Komolibus, K.; Piwonski, T.; Reyner, C. J.; Liang, B.; Huyet, G.; Huffaker, D. L.; Viktorov, E. A.; Houlihan, J. Absorption dynamics of type-II GaSb/GaAs quantum dots. Opt. Mater. Express 2017, 7, 1424.

(32) Lee, A. D.; Jiang, Q.; Tang, M.; Zhang, Y.; Seeds, A. J.; Liu, H. Y. InAs/GaAs Quantum-Dot Lasers Monolithically Grown on $\mathrm{Si}, \mathrm{Ge}$, and Ge-on-Si Substrates. IEEE J. Sel. Top. Quantum Electron. 2013, 19, 1901107.

(33) Tang, M.; Chen, S.; Wu, J.; Jiang, Q.; Dorogan, V. G.; Benamara, M.; Mazur, Y. I.; Salamo, G. J.; xSeeds, A.; Liu, H. Y. 1.3$\mu \mathrm{m}$ InAs/GaAs quantum-dot lasers monolithically grown on $\mathrm{Si}$ substrates using InAlAs/GaAs dislocation filter layers. Opt. Express 2014, 22, 11528 .

(34) Chen, S.; Li, W.; Wu, J.; Jiang, Q.; Tang, M.; Shutts, S.; Elliott, S. N.; Sobiesierski, A.; Seeds, A. J.; Ross, I.; Smowton, P. M.; Liu, H. Electrically pumped continuous-wave III-V quantum dot lasers on silicon. Nat. Photonics 2016, 10, 307.

(35) Wu, J.; Lee, A.; Jiang, Q.; Tang, M.; Seeds, A. J.; Liu, H. Electrically pumped continuous-wave $1.3-\mu \mathrm{m}$ InAs/GaAs quantum dot lasers monolithically grown on $\mathrm{Si}$ substrates. IET Optoelectron. 2014, 8, 20.

(36) Orchard, J. R.; Shutts, S.; Sobiesierski, A.; Wu, J.; Tang, M.; Chen, S.; Jiang, Q.; Elliott, S.; Beanland, R.; Liu, H. Y.; Smowton, P.; Mowbray, D. J. In situ annealing enhancement of the optical properties and laser device performance of InAs quantum dots grown on $\mathrm{Si}$ substrates. Opt. Express 2016, 24, 6196.

(37) Beanland, R. Dark field transmission electron microscope images of III-V quantum dot structures. Ultramicroscopy 2005, 102, 115.

(38) Teissier, R.; Sicault, D.; Harmand, J. C.; Ungaro, G.; Le Roux, G.; Largeau, L. J. Temperature-dependent valence band offset and 
band-gap energies of pseudomorphic GaAsSb on GaAs. J. Appl. Phys. 2001, 89, 5473.

(39) Guffarth, F.; Heitz, R.; Geller, M.; Kapteyn, C.; Born, H.; Sellin, R.; Hoffmann, A.; Bimberg, D.; Sobolev, N. A.; Carmo, M. C. Radiation hardness of InGaAs/GaAs quantum dots. Appl. Phys. Lett. 2003, 82, 1941.

(40) Young, R. J.; Stevenson, M. R.; Atkinson, P.; Cooper, K.; Ritchie, D. A.; Shields, A. J. Improved fidelity of triggered entangled photons from single quantum dots. New J. Phys. 2006, 8, 29.

(41) Young, M. P.; Woodhead, C. S.; Roberts, J.; Noori, Y. J.; Noble, M. T.; Krier, A.; Smakman, E. P.; Koenraad, P. M.; Hayne, M.; Young, R. J. Photoluminescence studies of individual and few $\mathrm{GaSb} / \mathrm{GaAs}$ quantum rings. $A I P A d v .2014,4,117127$.

(42) He, Y.-M.; He, Y.; Wei, Y.-J.; Wu, D.; Atatüre, M.; Schneider, C.; Höfling, S.; Kamp, M.; Lu, C.-Y.; Pan, J.-W. On-demand semiconductor single-photon source with near-unity indistinguishability. Nat. Nanotechnol. 2013, 8, 213.

(43) Vamivakas, A. N.; Zhao, Y.; Fält, S.; Badolato, A.; Taylor, J. M.; Atatüre, M. Nanoscale Optical Electrometer. Phys. Rev. Lett. 2011, 107, 166802.

(44) Houel, J.; Kuhlmann, A. V.; Greuter, L.; Xue, F.; Poggio, M.; Gerardot, B. D.; Dalgarno, P. A.; Badolato, A.; Petroff, P. M.; Ludwig, A.; Reuter, D.; Wieck, A. D.; Warburton, R. J. Probing Single-Charge Fluctuations at a GaAs/AlAs Interface Using Laser Spectroscopy on a Nearby InGaAs Quantum Dot. Phys. Rev. Lett. 2012, 108, 107401.

(45) Fox, A. M. Optical Properties of Solids; Oxford University Press, 2001; p 131.

(46) Fry, P. W.; Itskevich, I. E.; Mowbray, D. J.; Skolnick, M. S.; Finley, J. J.; Barker, J. A.; O’Reilly, E. P.; Wilson, L. R.; Larkin, I. A.; Maksym, P. A.; Hopkinson, M.; Al-Khafaji, M.; David, J. R. P.; Cullis, A. G.; Hill, G.; Clark, J. C. Inverted Electron-Hole Alignment in InAsGaAs Self-Assembled Quantum Dots. Phys. Rev. Lett. 2000, 84, 733.

(47) Lee, S. M.; Bajaj, K. K. A quantum statistical theory of linewidths of radiative transitions due to compositional disordering in semiconductor alloys. J. Appl. Phys. 1993, 73, 1788.

(48) Zrenner, A.; Butov, L. Y.; Hagn, M.; Abstreiter, G.; Bohm, G.; Weimann, G. Quantum dots formed by interface fluctuations in AlAs/ GaAs coupled quantum well structures. Phys. Rev. Lett. 1994, 72, 3382. 